\title{
FINITE SERIES AND COMPLETE SOLUTION OF $n$-ORDER $\alpha$-DIFFERENCE EQUATION
}

\author{
G. Britto Antony Xavier ${ }^{\S}$, P. Rajiniganth ${ }^{2}$ \\ ${ }^{1,2}$ Department of Mathematics \\ Sacred Heart College \\ Tirupattur, 635601, Vellore District \\ Tamil Nadu, INDIA
}

\begin{abstract}
In this paper, we define $\mathrm{n}^{\text {th }}$ order $\alpha$-difference operator and its equation and derive the corresponding discrete version of Leibnitz's and Montmorte's Theorems. Also, we investigate the numerical and complete solutions of $\mathrm{n}^{\text {th }}$ order $\alpha$-difference equation for finding the values of the sum of general partial sums of various finite series on arithmetic-geometric progression in the field of finite difference methods.
\end{abstract}

AMS Subject Classification: $39 \mathrm{~A} 70,47 \mathrm{~B} 39,39 \mathrm{~A} 10$

Key Words: generalized $\alpha$-difference equation, numerical solution, complete solution, generalized polynomial factorial

\section{Introduction}

In 1984, Jerzy Popenda[4] introduced a particular type of difference operator $\Delta_{\alpha}$ defined on $u(k)$ as $\Delta_{\alpha} u(k)=u(k+1)-\alpha u(k)$. In 1989 Miller and Rose[5] introduced the discrete analogue of the Riemann-Liouville fractional derivative and proved some properties of the fractional difference operator. The general fractional h-difference Riemann-Liouville operator and its inverse $\Delta_{h}^{-\nu} f(t)$ were mentioned in $[2,3]$. As application of $\Delta_{h}^{-\nu}$, by taking $\nu=m$ (positive integer) and $h=\ell$, the sum of $m^{\text {th }}$ partial sums on $n^{\text {th }}$ powers of arithmetic,

Received:

August 4, 2015

Revised: $\quad$ November 27, 2016

Published: December 11, 2016

${ }^{\S}$ Correspondence author (c) 2016 Academic Publications, Ltd. url: www.acadpubl.eu 
arithmetic-geometric progressions and products of $\mathrm{n}$ consecutive terms of arithmetic progression have been derived using $\Delta_{\ell}^{-m} u(k)[8]$.

In 2011, M. Maria Susai Manuel, et al, [9] have extended the definition of $\Delta_{\alpha}$ to $\Delta_{\alpha(\ell)}$ defined as $\Delta_{\alpha(\ell)} u(k)=u(k+\ell)-\alpha u(k)$ for the real valued function $u(k)$ and $\ell \in(0, \infty)$. In [10], the authors have used the generalized $\alpha$-difference equation;

$$
v(k+\ell)-\alpha v(k)=u(k), \quad k \in[0, \infty), \quad \ell \in(0, \infty),
$$

and obtained a numerical solution of the above equation in the form

$$
v(k)=\left.\Delta_{\alpha(\ell)}^{-1} u(k)\right|_{j} ^{k}=\sum_{r=1}^{\left[\frac{k}{\ell}\right]} \alpha^{r-1} u(k-r \ell), \quad j=k-\left[\frac{k}{\ell}\right] \ell .
$$

There are two type of solutions for the equation (1.1); one is numerical another one is closed form solution. If we are able to find a closed form solution of equation (1.1), which is coinciding with the numerical solution of that equation, then we can obtain formula for finding the values of several finite series. In this paper, we extend the theory and applications of $\Delta_{\alpha(\ell)}$ developed in [10] to generalized $n^{\text {th }}$ kind $\alpha$-difference equation mentioned below

$$
\begin{gathered}
v\left(k+\ell_{1}+\ell_{2}+\cdots+\ell_{n}\right)-\alpha\left\{v\left(k+\ell_{1}+\ell_{2}+\cdots+\ell_{n-1}\right)+\right. \\
v\left(k+\ell_{1}+\ell_{2}+\cdots+\ell_{n-2}+\ell_{n}\right)+v\left(k+\ell_{1}+\ell_{2}+\cdots+\ell_{n-3}+\ell_{n-1}+\ell_{n}\right)+\cdots+ \\
\left.v\left(k+\ell_{1}+\ell_{3}+\cdots+\ell_{n}\right)+v\left(k+\ell_{2}+\ell_{3}+\cdots+\ell_{n}\right)\right\}+\alpha^{2}\left\{v\left(k+\ell_{1}+\cdots+\ell_{n-2}\right)\right. \\
+v\left(k+\ell_{1}+\ell_{2}+\cdots+\ell_{n-3}+\ell_{n-1}\right)+\cdots+v\left(k+\ell_{1}+\ell_{3}+\cdots+\ell_{n-1}\right)+ \\
v\left(k+\ell_{2}+\ell_{3}+\cdots+\ell_{n-1}\right)+v\left(k+\ell_{1}+\ell_{2}+\cdots+\ell_{n-3}+\ell_{n}\right)+v\left(k+\ell_{1}+\ell_{2}+\right. \\
\left.\cdots+\ell_{n-4}+\ell_{n-2}+\ell_{n}\right)+\cdots+v\left(k+\ell_{2}+\ell_{3}+\cdots+\ell_{n-2}+\ell_{n}\right)+\cdots+v\left(k+\ell_{1}+\right. \\
\left.\left.\ell_{4}+\cdots+\ell_{n}\right)+v\left(k+\ell_{2}+\ell_{4}+\cdots+\ell_{n}\right)+v\left(k+\ell_{3}+\ell_{4}+\cdots+\ell_{n}\right)\right\} \\
+\cdots+(-\alpha)^{n-1}\left\{v\left(k+\ell_{1}\right)+\cdots+v\left(k+\ell_{n}\right)\right\}+(-\alpha)^{n} v(k)=u(k) .
\end{gathered}
$$

where $\alpha, \ell_{1}, \ell_{2}, \ell_{3}, \cdots, \ell_{n}$ are the positive reals and $k \geq 0$ is variable. By defining $\Delta_{\alpha\left(\ell_{1}, \ell_{2}, \cdots, \ell_{n}\right)}=\Delta_{\alpha\left(\ell_{3}\right)}\left(\Delta_{\alpha\left(\ell_{2}\right)}\left(\Delta_{\alpha\left(\ell_{1}\right)} \cdots\left(\Delta_{\alpha\left(\ell_{n}\right)} v(k)\right)\right)\right)=\Delta_{L_{n}}$, (1.3) can be expressed as

$$
\underset{L_{n}}{\Delta_{\alpha}} v(k) \equiv \sum_{t=0}^{n}(-\alpha)^{n-t} \sum_{A \in t\left(J_{n}\right)} v\left(k+\sum_{i \in A} \ell_{i}\right)=u(k)
$$


where $L_{n}=\left\{\ell_{1}, \ell_{2}, \ell_{3}, \cdots, \ell_{n}\right\}, J_{n}=\{1,2, \cdots, n\}$ and $0\left(J_{n}\right)=\phi$ (empty set), $1\left(J_{n}\right)=\{\{1\},\{2\}, \cdots,\{n\}\}, 2\left(J_{n}\right)=\{\{1,2\},\{1,3\}, \cdots,\{1, n\},\{2,1\},\{2,3\}$, $\cdots\{2, n\}, \cdots,\{n, 1\},\{n, 2\}, \cdots,\{n, n-1\}\}, \cdots, n\left(J_{n}\right)=\{1,2,3, \cdots, n\} . \quad$ In general $t\left(J_{n}\right)=$ set of all subsets of size $t$ from the set $J_{n}=\left\{\ell_{1}, \ell_{2}, \ldots, \ell_{n}\right\}$. We also investigate numerical - complete solutions of relation of (1.4) to obtain the values of the sum of general partial sums of several finite series. When $\alpha=1$, the results of this paper coincide with [8].

\section{Preliminaries}

Before stating and proving our results, we present some notations, basic definitions and preliminary results which will be used for further subsequent discussions. Let $\ell>0, k \in[0, \infty),\left[\frac{k}{\ell}\right]$ denotes the integer part of $\frac{k}{\ell}, j=$ $k-\left[\frac{k}{\ell}\right] \ell, E^{\ell} v(k)=v(k+\ell)$ and $\alpha \neq 0$.

The following lemmas can be obtained by simple algebraic derivations.

Lemma 2.1. Let $\ell_{1}, \ell_{2}, \cdots, \ell_{n}$ be positive reals. Then

$$
\begin{gathered}
\text { (i) } \begin{array}{c}
\Delta_{\alpha} \\
L_{n}
\end{array}=\sum_{t=0}^{n}(-\alpha)^{n-t} \sum_{A \in t\left(J_{n}\right)} E^{\sum_{i \in A} \ell_{i}} \\
\text { (ii) } \Delta_{L_{n}}=\sum_{t=0}^{n}(-\alpha)^{n-t} \sum_{A \in t\left(J_{n}\right)} \Delta_{\alpha\left(\sum_{i \in A} \ell_{i}\right)}+ \\
(-1)^{n} \alpha(\alpha-1)^{n}+(-1)^{n+1} \alpha^{n}(\alpha-1) . \\
\text { (iii) } \Delta_{L_{\alpha}}=\prod_{r=1}^{n} \sum_{s=1}^{\ell_{r}} \ell_{r} C_{s} \Delta^{s}-\alpha .
\end{gathered}
$$

Lemma 2.2. For the positive integer $r$,

$$
\begin{aligned}
& \text { (i) } \begin{array}{l}
\Delta_{\alpha}^{\mathrm{r}} \\
L_{n}
\end{array}=\prod_{t=1}^{n} \sum_{i=0}^{r}(-\alpha)^{i} r C_{i} E^{\ell_{t}(r-i)} . \\
& \text { (ii) } \Delta_{L_{n}}^{\mathrm{r}} u(k)=\prod_{t=1}^{n} \sum_{i=0}^{r}(-\alpha)^{i} r C_{i} u\left(k+\ell_{t}(r-i)\right) . \\
& \text { (iii) } \Delta_{\alpha}=\prod_{t=1}^{n}\left[\prod_{i=1}^{n}\left(\Delta_{\alpha\left(\ell_{t_{i}}\right)}+\alpha\right)-\alpha\right] .
\end{aligned}
$$


(iv) For the positive integer $n$,
(a) $\underset{n L_{n}}{\Delta_{\alpha}}=\sum_{t=0}^{n}(-\alpha)^{n-t} \sum_{A \in t\left(J_{n}\right)} E^{\sum_{i \in A} \ell_{i}}$
(b) $\underset{n L_{n}}{\Delta_{\alpha}}=\sum_{t=0}^{n}(-\alpha)^{n-t} \sum_{A \in t\left(J_{n}\right)}\left(\alpha+\Delta_{\alpha\left(\sum_{i \in A} \ell_{i}\right)}\right)^{n}$.
(c) $\underset{n L_{n}}{\Delta_{\alpha}}=\sum_{r=1}^{n} n C_{r} \alpha^{n-r} \sum_{t=0}^{n}(-\alpha)^{n-t} \sum_{A \in t\left(J_{n}\right)} \Delta_{\alpha\left(\sum_{i \in A} \ell_{i}\right)}^{r}$.
(d) $\Delta_{L_{n}}^{\mathrm{n}}=\prod_{t=1}^{n}\left(\sum_{i=0}^{n-1}(-\alpha)^{i} n C_{i} \Delta_{(n-i) \ell_{t}}\right)$.

Theorem 2.3. Let $u(k)$ and $v(k)$ be two real valued functions. Then

$$
\underset{L_{n}}{\Delta_{\alpha}^{\mathrm{m}}}[u(k) v(k)]=\sum_{r=0}^{m} m C_{r} \alpha^{m-r} \underset{\substack{\Delta_{n-\left\{\ell_{i}\right\}} \\ L_{\alpha\left(\ell_{i}\right)}}}{\mathrm{m}}\left[\Delta^{r}(k) \Delta_{\alpha\left(\ell_{i}\right)}^{m-r} v\left(k+r \ell_{i}\right)\right] .
$$

Proof. The proof follows from the generalized Leibnitz Theorem (Theorem $2.5,[10])$ and (1.4).

Lemma 2.4. If $n$ is a positive integer, then

$$
\begin{gathered}
\sum_{t=1}^{n} \sum_{A \in t\left(J_{n}\right)}(-\alpha)^{n-t} E^{n \sum_{i \in A} \ell_{i}}=\sum_{r_{1}=1}^{n} \sum_{r_{2}=0}^{n} \alpha^{r_{1}} n C_{r_{1}}(-\alpha)^{r_{2}} r_{1} C_{r_{2}} \\
\sum_{t=0}^{n} \sum_{A \in t\left(J_{n}\right)}(-\alpha)^{n-t} \Delta_{\alpha\left(r_{1}-r_{2}\right)\left(\sum_{i \in A} \ell_{i}\right)}
\end{gathered}
$$

Theorem 2.5. If $n$ and $p$ are the positive integers, then

$$
\begin{aligned}
& \sum_{t=1}^{n} \sum_{A \in t\left(J_{n}\right)}(-\alpha)^{n-t}\left(k+n \sum_{i \in A} \ell_{i}\right)^{p}=\sum_{r=0}^{n} \alpha^{n-r} n C_{r} \\
& \sum_{s=0}^{r-1} \sum_{t=0}^{n} r C_{s}(-\alpha)^{s+n-t} \sum_{A \in t\left(J_{n}\right)}\left(k+(r-s) \sum_{i \in A} \ell_{i}\right)^{p}
\end{aligned}
$$

Proof. The proof follows by operating (2.6) on $u(k)=k^{p}$.

Lemma 2.6. Let $u(k)$ be real valued function and $x$ is real. Then

$$
\sum_{j=0}^{\infty} \sum_{t=1}^{n} \sum_{A \in t\left(J_{n}\right)}(-\alpha)^{n-t} \frac{x^{j \sum_{i \in A} \ell_{i}} u\left(j \sum_{i \in A} \ell_{i}\right)}{j !\left(\sum_{i \in A} \ell_{i}\right)^{j}}
$$




$$
\begin{gathered}
=\sum_{t=1}^{n} \sum_{A \in t\left(J_{n}\right)}(-\alpha)^{n-t} e^{\frac{\sum_{i \in A} \ell_{i} E^{\sum_{i \in A} \ell_{i}}}{\sum_{i \in A} \ell_{i}}} u(0) \\
=\sum_{t=1}^{n} \sum_{A \in t\left(J_{n}\right)}(-\alpha)^{n-t} e^{\frac{\alpha x x_{i \in A} \ell_{i}}{\sum_{i \in A} \ell_{i}}} e^{\frac{\sum_{i \in A} \ell_{i} \Delta_{\alpha\left(\sum_{i \in A} \ell_{i}\right)}}{\sum_{i \in A} \ell_{i}}} u(0)
\end{gathered}
$$

Proof. The proof follows from (2.1), $u(k)=E^{k} u(0)$ and $E^{\ell}=\alpha+\Delta_{\alpha(\ell)}$.

Corollary 2.7. If $\ell$ is a positive real, then

$$
\begin{aligned}
\sum_{j=0}^{\infty} \sum_{t=1}^{n}(-\alpha)^{n-t} n C_{t} \frac{x^{j t \ell} u(j t \ell)}{j !(t \ell)^{j}} u(0)= & \sum_{t=1}^{n}(-\alpha)^{n-t} n C_{t} e^{\frac{x^{t \ell} E^{t \ell}}{t \ell)}} u(0) \\
& =\sum_{t=1}^{n}(-\alpha)^{n-t} n C_{t} e^{\frac{\alpha x^{t \ell}}{t \ell}} e^{\frac{x^{t \ell} \Delta_{\alpha(t \ell)}}{t \ell}} u(0)
\end{aligned}
$$

\section{Numerical - Complete Solutions of (1.3)}

In this section, we derive a numerical solution and a complete solution of the equation (1.3) and obtain the value of several finite series in the field of finite difference methods.

Definition 3.1. A function $v(k)$ satisfying $n^{\text {th }}$ order $\alpha$-difference equation (1.4) is called solution of that equation and is denoted as $v(k)=\underset{L_{n}}{-1} u(k)$.

Lemma 3.2. [7] If $s_{r}^{n}$ and $S_{r}^{n}$ are the Stirling numbers of the first and second kinds and $k_{\ell}^{(n)}=k(k-\ell)(k-2 \ell) \ldots(k-(r-1) \ell)$, then

$$
k_{\ell}^{(n)}=\sum_{r=1}^{n} s_{r}^{n} \ell^{n-r} k^{r} \quad \text { and } \quad k^{n}=\sum_{r=1}^{n} S_{r}^{n} \ell^{n-r} k_{\ell}^{(r)} .
$$

The proof of the following lemmas are trival.

Lemma 3.3. Let $0<\ell<k$. Then, when $\ell_{1}=\ell_{2}=\cdots=\ell_{n}$ in (1.3),

$$
v(k)=\sum_{r=n}^{\left[\frac{k}{\ell}\right]} \alpha^{r-n} \frac{[r-1]^{(n-1)}}{(n-1) !} u(k-r \ell)
$$


satisfies the generalized $n^{\text {th }}$ order $\alpha$-difference equation

$$
\sum_{r=0}^{n} n C_{r}(-\alpha)^{n-r} v(k+r \ell)=u(k) .
$$

Hence (3.2) is called as a numerical solution of the equation (3.3).

Lemma 3.4. If $v(k)$ is any closed solution of the equation (3.3), then

$$
\begin{aligned}
w_{n}(k)=v(k) & -\frac{1}{(n-1) !}\left[\frac{k}{\ell}\right]^{(n-1)} \alpha^{\left[\frac{k}{\ell}\right]-(n-1)} \Delta_{\alpha(\ell)}^{-1} v(j)-\frac{1}{(n-2) !}\left[\frac{k}{\ell}\right]^{(n-2)} \times \\
& \times \alpha^{\left[\frac{k}{\ell}\right]-(n-2)} \Delta_{\alpha(\ell, \ell)}^{-1} v(j)-\cdots-\alpha^{\left[\frac{k}{\ell}\right]} \Delta_{\alpha(\ell, \ell, \cdots, \ell)}^{-1} v(j),
\end{aligned}
$$

is also a solution of the equation (3.3). Now $w_{n}(k)$ is called as complete solution of the equation (3.3).

Lemma 3.5. Let $P_{k}$ be any function of $k$ on $[0, \infty)$, then

$$
\begin{aligned}
v(k)= & \left.\frac{\lambda^{k}}{\left(\lambda^{\ell}-\alpha\right)^{n}} \sum_{t=0}^{\infty}(-1)^{t} \frac{[t+(n-1)]^{(t)}}{(t) !}\left[\frac{\lambda^{\ell} \Delta_{\ell}}{\left(\lambda^{\ell}-\alpha\right)}\right]^{t} P_{k}\right|_{j} ^{k}-\sum_{i=1}^{n-1} \frac{\lambda^{j}}{\left(\lambda^{\ell}-\alpha\right)^{i}} \sum_{t=0}^{\infty} \times \\
& \left.\frac{[t+(i-1)]^{(t)}}{(t) !} \frac{\lambda^{t \ell} \Delta_{\ell}^{t}}{\left(\lambda^{\ell}-\alpha\right)^{t}} P_{j}\left\{\frac{1}{(n-i) !}\left[\frac{k}{\ell}\right]^{(n-i)} \alpha\left[\frac{k}{\ell}\right]-(n-i)\right\}\right|_{j} ^{k}
\end{aligned}
$$

is a complete solution of the equation (3.3), for $u(k)=\lambda^{k} P_{k}$.

Proof. From the generalized $\alpha$-difference operator, we find that

$\alpha) F_{k}$ (or) $F_{k}=\left(\lambda^{\ell} E^{\ell}-\alpha\right)^{-1} P_{k}$, yields

$$
\begin{aligned}
& \Delta_{\alpha(\ell)} \lambda^{k} F_{k}=\lambda^{k+\ell} F_{(k+\ell)}-\alpha \lambda^{k} F_{k} \\
& =\lambda^{k}\left(\lambda^{\ell} E^{\ell}-\alpha\right) F_{k}=\lambda^{k} P_{k}, \text { where } P_{k}=\left(\lambda^{\ell} E^{\ell}-\right.
\end{aligned}
$$

$$
\begin{aligned}
\Delta_{\alpha(\ell)}^{-1} \lambda^{k} P_{k} & =\lambda^{k} F_{k}=\lambda^{k}\left(\lambda^{\ell} E^{\ell}-\alpha\right)^{-1} P_{k} \\
& =\frac{\lambda^{k}}{\left(\lambda^{\ell}-\alpha\right)}\left[1-\frac{\lambda^{\ell}}{\left(\lambda^{\ell}-\alpha\right)} \Delta_{\ell}+\frac{\lambda^{2 \ell}}{\left(\lambda^{\ell}-\alpha\right)^{2}} \Delta_{\ell}^{2}+\ldots\right] P_{k}
\end{aligned}
$$

and applying the limit from $j$ to $k$ we obtain

$$
\left.\Delta_{\alpha(\ell)}^{-1} \lambda^{k} P_{k}\right|_{j} ^{k}=\left.\frac{\lambda^{k}}{\left(\lambda^{\ell}-\alpha\right)} \sum_{t=0}^{\infty}(-1)^{t}\left[\frac{\lambda^{\ell} \Delta_{\ell}}{\left(\lambda^{\ell}-\alpha\right)}\right]^{t} P_{k}\right|_{j} ^{k},
$$

which is a complete solution of the generalized first order $\alpha$ - difference equation $v(k+\ell)-\alpha v(k)=\lambda^{k} P_{k}$. Now taking $\Delta_{\alpha(\ell)}^{-1}$ in the above expression and taking limit from $j$ to $k$, we get

$$
\left.\Delta_{\alpha(\ell, \ell)}^{-1} \lambda^{k} P_{k}\right|_{j} ^{k}=\left.\frac{\lambda^{k}}{\left(\lambda^{\ell}-\alpha\right)^{2}} \sum_{t=0}^{\infty}(-1)^{t}(t+1)\left[\frac{\lambda^{\ell} \Delta_{\ell}}{\left(\lambda^{\ell}-\alpha\right)}\right]^{t} P_{k}\right|_{j} ^{k}-
$$




$$
\left.\frac{\lambda^{j}}{\left(\lambda^{\ell}-\alpha\right)} \sum_{t=0}^{\infty}(-1)^{t}\left[\frac{\lambda^{\ell} \Delta_{\ell}}{\left(\lambda^{\ell}-\alpha\right)}\right]^{t} P_{j}\left\{\left[\frac{k}{\ell}\right] \alpha{ }^{\left[\frac{k}{\ell}\right]-1}\right\}\right|_{j} ^{k}
$$

Continuing the same process upto $n$ times and taking limit from $j$ to $k$, we get (3.5).

Lemma 3.6. If $k \geq n \ell$, then $v(k)=\sum_{r=n}^{\left[\frac{k}{\ell}\right]} \alpha^{r-n} \frac{[r-1]^{(n-1)}}{(n-1) !} \lambda^{k-r \ell} P_{k-r \ell}$ is a numerical solution of (3.3) for the function $u(k)=\lambda^{k} P_{k}$.

Proof. From the inverse of generalized $\alpha$ - difference operator and taking the limit from $j$ to $k$, we find

$$
\left.\Delta_{\alpha(\ell)}^{-1} \lambda^{k} P_{k}\right|_{j} ^{k}=\sum_{r=1}^{\left[\frac{k}{\ell}\right]} \alpha^{r-1} \lambda^{k-r \ell} P_{k-r \ell}
$$

which is a numerical solution of the generalized first order $\alpha$ - difference equation $v(k+\ell)-\alpha v(k)=\lambda^{k} P_{k}$. Now taking $\Delta_{\alpha(\ell)}^{-1}$ in the above expression, applying the limit from $j$ to $k$ we get

$$
\left.\Delta_{\alpha(\ell, \ell)}^{-1} \lambda^{k} P_{k}\right|_{j} ^{k}=\sum_{r=2}^{\left[\frac{k}{\ell}\right]}(r-1) \alpha^{r-2} \lambda^{k-r \ell} P_{k-r \ell} .
$$

Continuing the same process upto $n$ times, applying the limit from $j$ to $k$ and using Lemma (3.3), we get the proof.

Theorem 3.7. Let $P_{k}$ be any function of $k$ on $[0, \infty)$. Then,

$$
\begin{gathered}
\sum_{r=n}^{\left[\frac{k}{\ell}\right]} \alpha^{r-n} \frac{[r-1]^{(n-1)}}{(n-1) !} \lambda^{k-r \ell} P_{k-r \ell}=\frac{\lambda^{k}}{\left(\lambda^{\ell}-\alpha\right)^{n}} \sum_{t=0}^{\infty}(-1)^{t} \times \\
\left.\frac{[t+(n-1)]^{(t)}}{(t) !}\left[\frac{\lambda^{\ell} \Delta_{\ell}}{\left(\lambda^{\ell}-\alpha\right)}\right]^{t} P_{k}\right|_{j} ^{k}-\sum_{i=1}^{n-1} \frac{\lambda^{j}}{\left(\lambda^{\ell}-\alpha\right)^{i}} \sum_{t=0}^{\infty} \frac{[t+(i-1)]^{(t)}}{(t) !} \\
\times\left.\left[\frac{\lambda^{\ell} \Delta_{\ell}}{\left(\lambda^{\ell}-\alpha\right)}\right]^{t} P_{j}\left\{\frac{1}{(n-i) !}\left[\frac{k}{\ell}\right]^{(n-i)} \alpha{ }^{\left[\frac{k}{\ell}\right]-(n-i)}\right\}\right|_{j} ^{k} .
\end{gathered}
$$

Proof. The proof follows by equating the lemma (3.5) and (3.6) 
The following theorem gives the formula for sum of $(n-1)^{\text {th }}$ partial sums of finite arithmetic-geometric series.

Theorem 3.8. Consider all the terms in Theorem 3.7. Then

$\sum_{r=n}^{\left[\frac{k}{\ell}\right]} \alpha^{r-n} \frac{[r-1]^{(n-1)}}{(n-1) !} \lambda^{k-r \ell}(k-r \ell)_{\ell}^{(n)}=\frac{\lambda^{k}}{\left(\lambda^{\ell}-\alpha\right)^{n}} \sum_{t=0}^{n}(-1)^{t} \times$

$\frac{[t+(n-1)]^{(t)}}{(t) !} \times\left. n^{(t)} \ell^{t} \frac{\lambda^{t \ell}}{\left(\lambda^{\ell}-\alpha\right)^{t}} k_{\ell}^{(n-t)}\right|_{j} ^{k}-\sum_{i=1}^{n-1} \frac{\lambda^{j}}{\left(\lambda^{\ell}-\alpha\right)^{i}} \sum_{t=0}^{n}(-1)^{t} \frac{[t+(i-1)]^{(t)}}{(t) !} \times$

$$
\times\left.\frac{\lambda^{t \ell}}{\left(\lambda^{\ell}-\alpha\right)^{t}} n^{(t)} \ell^{t} j_{\ell}^{(n-t)} \frac{1}{(n-i) !}\left\{\left[\frac{k}{\ell}\right]^{(n-i)} \alpha{ }^{\left[\frac{k}{\ell}\right]-(n-i)}\right\}\right|_{j} ^{k}
$$

Proof. The proof follows by taking $P_{k}=k_{\ell}^{(n)}$ in Theorem (3.7).

The following example is illustrate of Theorem 3.8 when $n=3$.

Example 3.9. In (3.8), by taking $n=4$, we obtain

$$
\begin{gathered}
\sum_{r=4}^{\left[\frac{k}{\ell}\right]} \alpha^{r-4} \frac{[r-1]^{(3)}}{(3) !} \lambda^{k-r \ell}(k-r \ell)_{\ell}^{(4)}=\frac{\lambda^{k}}{\left(\lambda^{\ell}-\alpha\right)^{4}} \sum_{t=0}^{4}(-1)^{t} \frac{[t+(3)]^{(3)}}{3 !} \times \\
\times 4^{(t)} \ell^{t} \frac{\lambda^{t \ell}}{\left(\lambda^{\ell}-\alpha\right)^{t}} k_{\ell}^{\left.(4-t)\right|_{j} ^{k}}-\sum_{i=1}^{3} \frac{\lambda^{j}}{\left(\lambda^{\ell}-\alpha\right)^{i}} \sum_{t=0}^{4}(-1)^{t} \frac{[t+(i-1)]^{(t)}}{(t) !} \frac{\lambda^{t \ell}}{\left(\lambda^{\ell}-\alpha\right)^{t}} \times \\
\times\left. 4^{(t)} \ell^{t} j_{\ell}^{(4-t)} \frac{1}{(4-i) !}\left\{\left[\frac{k}{\ell}\right]^{(4-i)} \alpha^{\left[\frac{k}{\ell}\right]-(4-i)}\right\}\right|_{j} ^{k}
\end{gathered}
$$

In particular, $k=31, \ell=3, \alpha=5, \lambda=3$ and $j=1$, we get,

$$
\begin{gathered}
\sum_{r=4}^{10} \frac{(r-1)^{(3)}}{3 !} 5^{r-4} 3^{31-3 r}(31-3 r)_{3}^{(4)}=\left.\sum_{t=0}^{3}(-1)^{t} \frac{(t+3)^{(3)}(3)^{(t)}}{3 !} \frac{3^{t} 3^{31+3 t} 31_{3}^{(3-t)}}{\left(3^{3}-5\right)^{t+3}}\right|_{1} ^{31} \\
-\left.\sum_{t=0}^{3}(-1)^{t}(t+2)^{(t)}(3)^{(t)} \frac{3^{t} 3^{31+3 t} 31_{3}^{(4-t)}}{\left(3^{3}-5\right)^{t+3}}\right|_{1} ^{31}-\sum_{t=0}^{3}(-1)^{t}(t+1) 3^{(t)} 3^{t} \frac{3^{1+t \ell}}{\left(3^{3}-5\right)^{t+2}} \\
\left.1_{3}^{(4-t)}\left\{\left[\frac{k}{3}\right] 5^{\left[\frac{k}{3}\right]-1}\right\}\right|_{1} ^{31}-\frac{1}{2} \sum_{t=0}^{3}(-1)^{t} 3^{(t)} \ell^{t} \frac{3^{1+3 t}}{\left(3^{3}-5\right)^{t+1}} 1_{3}^{(4-t)} \\
\left.\left\{\left[\frac{k}{3}\right]^{(2)} 5^{\left[\frac{k}{3}\right]-2}\right\}\right|_{1} ^{31}=59957519486040 .
\end{gathered}
$$

Following is the formula for sum $(n-1)^{\text {th }}$ partial sums of higher powers of arithmetic-geometric series.

Theorem 3.10. Consider all the terms in Theorem 3.7. Then, $\sum_{r=n}^{\left[\frac{k}{\ell}\right]} \frac{[r-1]^{(n-1)}}{(n-1) !} \alpha^{r-n} \lambda^{k-r \ell}(k-r \ell)^{n}=\frac{\lambda^{k \ell}}{\left(\lambda^{\ell}-\alpha\right)^{n}} \sum_{t=0}^{n}(-1)^{t} \frac{[t+(n-1)]^{(t)}}{(t) !} \times$ 


$$
\begin{aligned}
& \left.\frac{n^{(t)} \ell^{t} \lambda^{t \ell}}{\left(\lambda^{\ell}-\alpha\right)^{t}} \sum_{i=1}^{n-t} S_{i}^{n-t} \ell^{n-t-i} k_{\ell}^{(i)}\right|_{j} ^{k}-\sum_{i=1}^{n-1} \frac{\lambda^{j}}{\left(\lambda^{\ell}-\alpha\right)^{i}} \sum_{t=0}^{n}(-1)^{t} \frac{[t+(i-1)]^{(t)}}{(t) !} \times \\
& \left.\frac{n^{(t)} \ell^{t} \lambda^{t \ell}}{\left(\lambda^{\ell}-\alpha\right)^{t}} \sum_{i=1}^{n-t} S_{i}^{n-t} \ell^{n-t-i} j_{\ell}^{(i)}\left\{\frac{1}{(n-i) !}\left[\frac{k}{\ell}\right]^{(n-i)} \alpha\left[\frac{k}{\ell}\right]-(n-i)\right\}\right|_{j} ^{k}
\end{aligned}
$$

Proof. Taking $P_{k}=k^{n}$ in (3.8) and using (3.1), we get proof.

The following illustrates Theorem 3.10.

Corollary 3.11. If $\alpha \neq a^{\ell}, k \in[\ell, \infty)$ and $j=k-\left[\frac{k}{\ell}\right] \ell$, then $\sum_{r=n}^{\left[\frac{k}{\ell}\right]}$

$$
\begin{aligned}
& \frac{[r-1]^{(n-1)}}{(n-1) !} \alpha^{r-n}(k-r \ell) a^{k-r \ell}=\frac{k a^{k}}{\left(a^{\ell}-\alpha\right)^{n}}-\frac{n \ell a^{k+\ell}}{\left(a^{\ell}-\alpha\right)^{n+1}}-\sum_{i=1}^{n-1} \times \\
& \left.\left\{\frac{j a^{j}}{\left(a^{\ell}-\alpha\right)^{i}}-\frac{i \ell a^{j+\ell}}{\left(a^{\ell}-\alpha\right)^{i+1}}\right\}\left\{\frac{1}{(i-1) !}\left[\frac{k}{\ell}\right]^{(n-i)} \alpha{ }^{\left[\frac{k}{\ell}\right]-(n-i)}\right\}\right|_{j} ^{k}
\end{aligned}
$$

Proof. The proof follows by taking $n=1$ and $\lambda=a$ in (3.11).

Example 3.12. Taking $k=34, \ell=3, a=7, \alpha=3$ and $j=1$ in (3.12), we obtain

$$
\begin{gathered}
\sum_{r=4}^{\left[\frac{k}{3}\right]} \frac{(r-1)^{(3)}}{3 !} 3^{r-4}(k-3 r) 7^{k-3 r}=\left.\left\{\frac{k 7^{k}}{(340)^{3} 4}-\frac{4.3 .7^{k+3}}{(340)^{5}}\right\}\right|_{1} ^{34}-\sum_{i=1}^{4}\left\{\frac{7}{(340)^{i}}-\right. \\
\left.\frac{i .3 .7^{4}}{340^{i+1}}\right\}\left.\left\{\frac{1}{i-1}\left[\frac{k}{3}\right]^{4-i} 3^{\left[\frac{k}{3}\right]-(4-i)}\right\}\right|_{1} ^{34}=88748631141432943604 .
\end{gathered}
$$

The following theorem illustrates Theorem 3.8 for $\lambda=1$.

Theorem 3.13. Let $k \in[\ell, \infty)$ and $m$ is a positive integer and $\alpha \neq 1$. Then,

$$
\begin{gathered}
\sum_{r=m}^{\left[\frac{k}{\ell}\right]} \frac{[r-1]^{(m-1)}}{(m-1) !} \alpha^{r-m}(k-r \ell)_{\ell}^{(m)}=\left.\sum_{i=0}^{m} \frac{(-1)^{i}[i+(m-1)]^{(m-1)} m^{(i)} \ell^{i} k_{\ell}^{(m-i)}}{(m-1) !(1-\alpha)^{i+m}}\right|_{j} ^{k}-\sum_{r=1}^{m-1} \\
\left.\sum_{i=0}^{m} \frac{(-1)^{i} m^{(i)} \ell^{i} j_{\ell}^{(m-i)}}{(1-\alpha)^{i+r}(r-1) !} \frac{[i-(r-1)]^{(r-1)}}{(r-1) !}\left\{\left[\frac{k}{\ell}\right]^{(m-r)} \alpha{ }^{\left[\frac{k}{\ell}\right]-(m-r)}\right\}\right|_{j} ^{k}
\end{gathered}
$$

Proof. The proof follows by taking $\lambda=1$ in Theorem 3.8 .

Corollary 3.14. Let $k \in[\ell, \infty), j=k-\left[\frac{k}{\ell}\right] \ell$ and $m=4$. Then

$$
\begin{gathered}
\sum_{r=4}^{\left[\frac{k}{\ell}\right]} \frac{[r-1]^{(3)}}{3 !} \alpha^{r-4}(k-r \ell)_{\ell}^{(4)}=\left.\sum_{i=0}^{4} \frac{(-1)^{i}[i+3]^{(3)} 4^{(i)} \ell^{i} k_{\ell}^{(4-i)}}{3 !(1-\alpha)^{i+4}}\right|_{j} ^{k}-\sum_{r=1}^{3} \sum_{i=0}^{4} \times \\
\left.\frac{(-1)^{i} 4^{(i)} \ell^{i} j_{\ell}^{(4-i)}}{(1-\alpha)^{i+r}(r-1) !} \frac{[i-(r-1)]^{(r-1)}}{(4-r) !}\left\{\left[\frac{k}{\ell}\right]^{(4-r)} \alpha{ }^{\left[\frac{k}{\ell}\right]-(4-r)}\right\}\right|_{j} ^{k}
\end{gathered}
$$


Theorem 3.15. Consider all the terms in Theorem 3.7. Then

$\sum_{r=4}^{\left[\frac{k}{\ell}\right]}$

$$
\begin{array}{r}
\frac{(r-1)^{(3)}}{3 !} \alpha^{r-4}(k-r \ell)^{n}=\left.\sum_{t=0}^{n} \frac{(-1)^{t}(t+3)^{(3)} n^{(t)}}{6(1-\alpha)^{t+4}} \sum_{i=1}^{n-t} S_{i}^{n-t} \ell^{n-i} k_{\ell}^{(i)}\right|_{j} ^{k} \\
-\sum_{m=0}^{n-1} \sum_{t=0}^{n} \frac{(-1)^{t}(t+(n-1))^{(t)} \ell^{t} n^{(t)}}{t !(1-\alpha)^{t+m}} \\
\left.\sum_{i=1}^{n-t} S_{i}^{n-t} \ell^{n-i} j_{\ell}^{(i)}\left\{\frac{1}{(n-m) !}\left[\frac{k}{\ell}\right]^{(n-m)} \alpha{ }^{\left[\frac{k}{\ell}\right]-(n-m)}\right\}\right|_{j} ^{k}
\end{array}
$$

Proof. Taking $P_{k}=k^{n}$ and $\lambda=1$ in (3.11), we get proof.

The following corollary illustrates Theorem 3.15.

Corollary 3.16. Let $k \in[\ell, \infty), \alpha \neq 1, n=4$ and $j=k-\left[\frac{k}{\ell}\right] \ell$. Then

$$
\begin{aligned}
\sum_{r=4}^{\left[\frac{k}{\ell}\right]} \frac{(r-1)^{(3)}}{3 !} \alpha^{r-4}(k-r \ell)^{4}=\left.\sum_{t=0}^{4} \frac{(-1)^{t}(t+3)^{(t)} 4^{(t)}}{(1-\alpha)^{t+4}} \sum_{i=1}^{4-t} S_{i}^{4-t} \ell^{4-i} k_{\ell}^{(i)}\right|_{j} ^{k} & -\sum_{m=0}^{3} \sum_{t=0}^{4} \frac{\left.(-1)^{t}(t+3)^{(} t\right) \ell^{t} 4^{(t)}}{t !(1-\alpha)^{t+m}} \\
& \left.\sum_{i=1}^{4-t} S_{i}^{4-t} \ell^{4-i} j_{\ell}^{(i)}\left\{\frac{1}{(4-m) !}\left[\frac{k}{\ell}\right]^{(4-m)} \alpha^{\left[\frac{k}{\ell}\right]-(4-m)}\right\}\right|_{j} ^{k} .
\end{aligned}
$$

Proof. The proof follows by taking $n=4$ in (3.15).

The following example is an illustration of Corollary 3.16.

Example 3.17. Taking $k=1001, \ell=100, \alpha=7$ and $j=1$ in (3.16), we obtain

$$
\begin{gathered}
\sum_{r=4}^{\left[\frac{k}{100}\right]} \frac{(r-1)^{(3)}}{3 !} 7^{r-4}(k-100 r)^{4}=\left.\sum_{t=0}^{4} \frac{(-1)^{t}(t+3)^{(3)} 4^{(t)}}{(-6)^{t+4}} \sum_{i=1}^{4-t} S_{i}^{4-t} 4^{4-i} k_{4}^{(i)}\right|_{1} ^{1001} \\
-\left.\sum_{m=0}^{3} \sum_{t=0}^{4} \frac{(-1)^{t}(t+3)^{(t)} \ell^{t} 4^{(t)}}{t !(-6)^{t+m}} \sum_{i=1}^{4-t} S_{i}^{4-t} \ell^{4-i} j_{\ell}^{(i)}\left\{\frac{1}{(4-m) !}\left[\frac{1001}{100}\right]^{(4-m)} 7^{\left[\frac{1001}{100}\right]-(4-m)}\right\}\right|_{1} ^{1001} \\
=22350256175871439660998266 .
\end{gathered}
$$

\section{Conclusion}

Here we derived the complete and Numerical solution of certain type of $n^{t h}$ order $\alpha$-difference equation and obtained several formulas on general partial sums of higher powers of A.G. progression. 


\section{Acknowledgments}

Research Supported by University Grants Commission, SERO, Hyderabad, India.

\section{References}

[1] R.P Agarwal, Difference Equations and Inequalities, Marcel Dekker, New York, 2000.

[2] N. R. O. Bastos, R. A. C. Ferreira, and D. F. M. Torres, Discrete-Time Fractional Variational Problems, Signal Processing, vol.91,no. 3,pp. 513-524, 2011.

[3] R. A. C. Ferreira and D. F. M. Torres, Fractional h-difference equations arising from the calculus of variations, Applicable Analysis and Discrete Mathematics, 5(1) (2011), 110-121.

[4] Jerzy Popenda and Blazej Szmanda, On the Oscillation of Solutions of Certain Difference Equations, Demonstratio Mathematica, XVII(1), (1984), 153 - 164.

[5] K.S.Miller and B.Ross, Fractional Difference Calculus in Univalent Functions, Fractional Calculus and Their Applications(Koriyama, 1988),pp . 139-152, Horwood, Chichester, UK, 1989.

[6] Ronald E.Mickens, Difference Equations, Van Nostrand Reinhold Company, New York, 1990.

[7] M.Maria Susai Manuel, G.Britto Antony Xavier and E.Thandapani, Theory of Generalized Difference Operator and Its Applications, Far East Journal of Mathematical Sciences, 20(2) (2006), 163 - 171.

[8] M. Maria Susai Manuel, G.Britto Antony Xavier, V. Chandrasekar, R. Pugalarasu and S. Elizabeth, Generalized Difference Operator of the Third kind and On Second Partial Sums of Products of Consecutive Terms of Arithmetic and Arithmetic-Geometric Progressions, International Journal of Pure and Applied Mathematics, 53(1) (2009), 109-120.

[9] M.Maria Susai Manuel, V.Chandrasekar and G.Britto Antony Xavier, Solutions and Applications of Certain Class of $\alpha$-Difference Equations, International Journal of Applied Mathematics, 24(6) (2011), 943-954.

[10] M.Maria Susai Manuel, V.Chandrasekar and G.Britto Antony Xavier, Theory of Generalized $\alpha$-Difference Operator and its Applications in Number Theory, Advances in Differential Equations and Control Processes, 9(2) (2012), 141-155. 
